\title{
Integrins: A flexible platform for endothelial vascular tyrosine kinase receptors
}

\author{
Lucia Napione $^{\mathrm{a}, \mathrm{b}}$, Ilaria Cascone ${ }^{\mathrm{a}, \mathrm{b}}$, Stefania Mitola ${ }^{\mathrm{c}}$, \\ Guido Serini $^{\mathrm{a}, \mathrm{b}}$, Federico Bussolino ${ }^{\mathrm{a}, \mathrm{b}, *}$ \\ a Dipartimento di Scienze Oncologiche, Università di Torino, 10060 Candiolo, Italy \\ b Istituto per la Ricerca e la Cura del Cancro, Università di Torino, 10060 Candiolo, Italy \\ ${ }^{\mathrm{c}}$ Dipartimento di Scienze Biomediche e Biotecnologie, Università di Brescia, 25100 Brescia, Italy
}

Available online 3 April 2007

\begin{abstract}
Compared to lower metazoans, vertebrates built up an exclusively new set of adhesion-related genes involved in the tissue development and in their functions. They include a large variety of extracellular matrix proteins and their heterodimeric integrin adhesive receptors. Integrins control the adhesive state of the cell through complex molecular mechanisms. Outside-in signalling informs the cell about the extracellular matrix environment, while Inside-out signalling results in changes in integrin functional activity. In the last 10 years it has well established a reciprocal integration of signals originating from integrins and receptors for soluble growth factors. This review summarizes the current understanding of this connection in vascular endothelial cells and highlights how integrins regulate a genetic program triggered by angiogenic inducers during embryo development and in adult life.

(c) 2007 Elsevier B.V. All rights reserved.
\end{abstract}

Keywords: Angiopoietin; Endothelium; Integrin; Semaphorin; VEGF

\section{Contents}

1. Introduction . . . . . . . . . . . . . . . . . . . . . . . . . . 18

2. Integrin-dependent modulation of VEGFRs. . . . . . . . . . . . . . . . . . . . . . . . 19

3. $\alpha 5 \beta 1$ integrin sustains Tie-2 activation . . . . . . . . . . . . . . . . . . . . . 20

4. Concluding remarks . . . . . . . . . . . . . . . . . . . . . . . . . 21

Acknowledgements. . . . . . . . . . . . . . . . . . . . . . . . 21

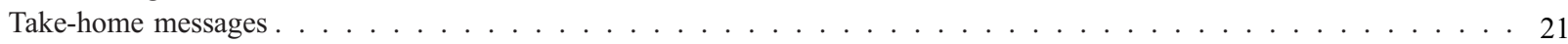

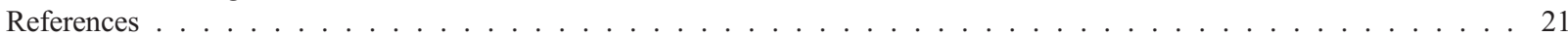

* Corresponding author. Dipartimento di Scienze Oncologiche Strada Provinciale 142, Km 3.9510060 Candiolo (TO) Italy. Tel.: +39 09119933347; fax: + 390119933524.

E-mail address: federico.bussolino@unito.it (F. Bussolino).

\section{Introduction}

The generation of a vascular system hierarchically organized into arteries, capillaries, and veins allows the 
efficient transport of oxygen and nutrients to the tissues so that they can growth, develop, and function. The complexity of these mechanisms occurring in embryonic and adult life is de-regulated in pathological settings including cancer, chronic inflammatory diseases, and retinopathies. In these conditions vasculature is highly chaotic and inefficient, mainly due to an unbalance between activating and inhibiting cues targeting vascular cells.

Five biological phases of angiogenesis have been established and characterized by different, overlapping genetic programs. Initiation is characterized by an endothelial cell (EC) shape change and increased permeability; progression phase includes the degradation of extracellular matrix, migration and proliferation of ECs; in the differentiation phase ECs stop to growth, survive in sub-optimal conditions, and differentiate into primitive capillaries; maturation phase includes the formation of new matrix and the recruitment of pericytes; remodeling phase generates the mature and hierarchically shaped vascular bed and required regression of some vessels by apotosis and pruning and branching of others [1]. Besides several soluble factors (PDGFs, TGFs, FGFs, HGF, chemokines, autacoids, cytokines), which appear to have a relevant but redundant role, the above steps are largely regulated by molecules with a high specificity for the vascular system: the family of vascular endothelium growth factors (VEGF) and their tyrosine kinase receptors (TKR), VEGFR-1, -2, and -3 , and the family of angiopoietins (Ang) and Tie-2 TKR [2,3].

It appears that all through the angiogenic process blood vessel ECs undergo collective movements regulated by several guidance cues that dynamically modulate their cell-to-cell and cell-to-extracellular matrix (ECM) adhesive contacts [4]. During tissue morphogenesis, while cell-to-cell interactions maintain the cohesion of multicellular structures, the finely tuned regulation of cell adhesion to the ECM controls the direction of collective cell motility. Integrin $\alpha \beta$ heterodimers are major ECM receptors that exist in different functional conformations with regard to their affinity for ligands [5] and play key roles in embryonic and post-natal angiogenesis [4].

In this review we will summarize data indicating that different integrins modulate the activity of VEGF receptors and Tie- 2 resulting in an additional control of EC functions during angiogenesis.

\section{Integrin-dependent modulation of VEGFRs}

During angiogenesis, ECs adhere to a provisional $\mathrm{ECM}$ through $\alpha \mathrm{v} \beta 3$ integrin. Once engaged with vitronection or fibrin, this integrin participates in a complex containing VEGFR-2 and PI 3-kinase [6-8]. The impact of the integrin pair on this complex is illustrated by studies performed with an anti- $\beta \quad 3$ antibody that interferes with $\alpha \mathrm{v} \beta 3$ clustering but not with cell adhesion to the ECM. Not only does the antibody perturb the formation of this complex, but it also markedly inhibits VEGFR-2-mediated phosphorylation, PI 3-kinase activity, focal adhesion dynamics as well as the proliferation and migration of ECs triggered by VEGF-A [6]. In contrast, $\alpha v \beta 3$ clustering is permissive for VEGFR-2 activation and an optimal response of ECs to VEGF-A $[6,8,9]$. Formation of the VEGFR-2/ $\alpha v \beta 3$ complex requires the extracellular domain and the $Y$ phosphorylation sites in the cytosol tail of $\beta 3$ integrin $[7,8]$. Because VEGF-A induces $Y$ phosphorylation of $\beta 3$ subunit though VEGFR-2 resulting in inside-out integrin activation $[7,8,10]$, it is evident that that these two receptors are able to crossactivate each other, therefore forming a functional partnership that is essential for successful angiogenesis.

The key role of VEGFR-2/ $\alpha \mathrm{v} \beta 3$ integrin complex has been further envisaged by the elucidation of the angiogenic effect of Factor XIII (FXIII), the transglutaminase that participates at the final step of the coagulation cascade. Activated FXIII crosslinks $\beta 3$ integrin to VEGFR-2 as well as enhances the noncovalent complex formation between the $\beta 3$ integrin and VEGFR-2. In this complex VEGFR-2 results to be activated in a VEGF-A - independent manner but requires both the tranglutaminase and TK activities of FXIII and VEGFR-2 [11].

In contrast to $\alpha \mathrm{v} \beta 3 /$ vitronectin (or fibrin) pair, which shows a permissive effect on VEGFR-2 phosphorylation, collagen I, the ligand of $\alpha 1 \beta 3$ and $\alpha 2 \beta 1$ integrins, exerts an inhibitory action on this TKR [12]. EC adhesion to collagen I reduces VEGF-A-induced VEGFR-2 autophosphorylation by recruiting the tyrosine phosphatase SHP2 to the pY117 of cytosolic tail of the receptor. The interaction of SHP2 to VEGFR-2 is strictly dependent on EC adhesion to collagen I and not to other ECM proteins. The more pronounced VEGFR-2 dephosphorylation is connected with a higher degree of its internalization. The down-regulation of VEGFR-2 by SHP2 could be considered a simpler and faster way to modify its behaviour and functions as compared to clathrin-mediated or caveolae-dependent endocytosis or to proteasome-mediated degradation [2]. The effect of collagen I to limit VEGFR-2 parallels the effect TIMP2, which negatively regulates VEGFR-2 by SHP1 activation [13]. In ECs stimulated with TIMP-2, SHP1 shifts from $\alpha_{3} \beta_{1}$ integrin to VEGFR-2, which is 
dephosphorylated and unable to signal a proliferative outcome. Similarly $\alpha_{1} \beta_{1}$ integrin engaged by collagen activates the T-cell protein tyrosine phosphatase function that inhibits EGF receptor signalling [14]. It has been reported that in vascular smooth muscle cells $\beta 3$ engagement by vitronectin results in tyrosine phosphorylation of its cytosolic domain and recruitment of SHP2, which modulates insulin growth factor I receptor[15]. Thus, we hypothesize a protective role on VEGFR-2 signalling by vitronectin-engaged $\alpha_{\mathrm{v}} \beta_{3}$, which recruits SHP2 and preserves the receptor from phosphatase activity. In contrast EC adhesion on collagen I, which does not dependent on $\alpha_{\mathrm{v}} \beta_{3}$ integrin, could allow SHP2 interacting with VEGFR-2.

The modulatory role of integrins on VEGFRs is not restricted to the type 2. VEGFR-3 stimulated by VEGF$\mathrm{C}$ or VEGF-D plays a major role in lymphangiogenesis. It has been demonstrated that VEGF-C - stimulated VEGFR-3 selectively associates with $\alpha 5 \beta 1$. Integrin $\alpha 5 \beta 1$ ligation by fibronectin is required for the optimal activation of VEGFR-3 signalling not only at the receptor level but also at its downstream effectors [16].

\section{3. $\alpha 5 \beta 1$ integrin sustains Tie-2 activation}

The angiopoietin family and its TKR Tie-2 have primary roles in the latter stages of angiogenesis where they control remodelling and stabilization of vessels. Ang-1 is required for correct organization and maturation of newly formed vessels, and promotes quiescence and structural integrity of adult vasculature [3].

We have recently reported that in ECs, Tie- 2 is present in free-form and associated with $\alpha 5 \beta 1$ integrin (Fig. 1, A, B). The activation of $\alpha 5 \beta 1$ integrin by fibronectin increases the complex with Tie-2 and modulates the time and concentration window of the receptor activation. When $\alpha 5 \beta 1$ is activated, Tie- 2 is phosphorylated at lower Ang-1 concentrations than those required on other ECM proteins. Furthermore, $\alpha 5 \beta 1 /$ Tie- 2 complex allows the TKR stimulation prolonged up to a hour, while the signal of free Tie-2 is more transient. Therefore, it seems that $\alpha 5 \beta 1$ activation could influence Tie-2 signal duration and signal strength. Ang- 1 does not modify the features of association between Tie-2 and fibronectin-engaged $\alpha 5 \beta 1$, but triggers biochemical signals that recruit $\mathrm{p} 85$ and focal adhesion kinase (FAK) to the complex. It is known that p85 binds activated Tie-2 [3], whereas FAK is recruited to the cytosolic tail of clusterized integrins at the focal adhesions [4]. Thus, Ang-1 stimulation mediates Tie-2 and $\alpha 5 \beta 1$ signalling, and allows a cross-talk between these pathways by acting at the level of Tie- $2 / \alpha 5 \beta 1$ complex. We demonstrated that Ang-1/Tie-2 activates integrins through the PI3-kinase signalling, which suggests that FAK recruitment to Tie- $2 / 5 \beta 1$ complex could be dependent on activated Tie-2 inside-out
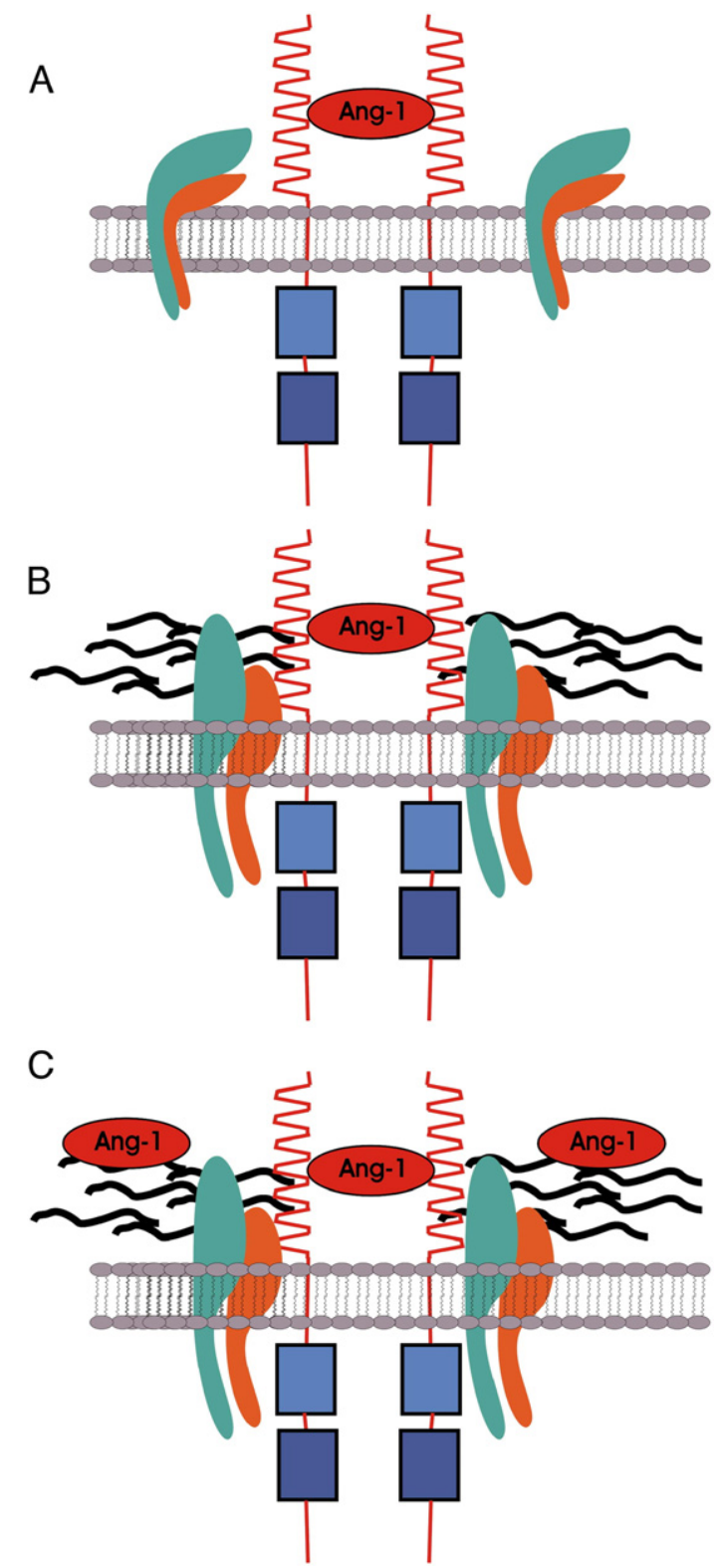

Fig. 1. Integrin $5 \beta 1$ modulates Tie-2 activity. (A) Free-Tie-2 responds to high Ang-1 concentrations with transient activation. (B) Integrin $5 \beta 1$ activated by fibronectin forms a complex with Tie-2, which in turn responds to a low Ang-1 concentration for long-lasting time; Tie-2 stimulation supports an integrin inside-out signal. (C) The signal coming from $5 \beta 1 /$ Tie- 2 complex is further reinforced by a direct $5 \beta 1$ integrin stimulation by Ang-1. This dual stimulation results in a combined activity of inside-out and outside-in signals (for details see text) 
signalling. Conversely, the observation that immobilized [17] or soluble Ang-1 binds and activates $\alpha 5 \beta 1$ in the absence of Tie- 2 demonstrates that Ang-1 promotes $\alpha 5 \beta 1$ outside-in signalling. All together the results suggest a model in which the synergism of Tie- $2 / \alpha 5 \beta 1$ inside-out/outside-in signalling would allow the stabilization of the complex and the activation of Tie-2 at lower Ang-1 concentrations (Fig. 1C).

\section{Concluding remarks}

Substantial evidence suggests that signalling is a compartmentalized event. Here we reported some examples describing how integrin adhesive receptors form supramolecular complexes with TKR in ECs localized at adhesive sites and influence the cell response to angiogenic inducers. These complexes described in ECs may represent a general strategy that contributes to the relative complexity of some species, including humans, compared to other organisms. Rather than using a larger number of genes to achieve a greater level of biological complexity higher organisms have evolved more intricate biochemical architectures, which are built from a relatively limited number of proteins. Future experiments will be required to understand how $\mathrm{TKR} /$ integrin complexes participate in EC differentiation during development and their behaviour in pathological angiogenesis.

\section{Acknowledgements}

Supported by Telethon Italy — grant no. GGP04127, Sixth Framework Programme of European Union Contract LSHM-CT-2003-503254, the "Associazione Italiana per la Ricerca sul Cancro", "Regione Piemonte", "M.I.U.R" and "Ministero della Salute".

\section{Take-home messages}

- Angiogenesis, a critical component of neoplastic and chronic inflammatory disorders, depends on the cooperation of growth factors and cell adhesion events.

- Integrin adhesive receptors form supramolecular complexes with TKRs in endothelial cell influencing cells response to angiogenic factors and blood vessel permeability.

- The formation of VEGFR2/ $\alpha \mathrm{v} \beta 3$ complex is permissive for an optimal TKRs activation, while clustering and activation of other integrin, such as $\alpha 1 \beta 3$ and $\alpha 2 \beta 3$, exerts an inhibitory action on
VEGFR2 by recruiting the tyrosine phosphatase SHP2.

- Ang-1binds and activates both Tie-2 and $\alpha 5 \beta 1$ allowing a synergism their inside-out/outside-in signalling.

- $\alpha 5 \beta 1 /$ Tie-2 complex stabilizes and extends the signal induced by Ang-1 compared to that of Tie- 2 alone.

- Also lymph angiogenesis, an important process involved in several normal and pathological conditions such as wound healing, inflammation or metastasis formation in several malignancies, can be modulated by integrins recruitment. Indeed $\alpha 5 / \beta 1$ binds and modulates VEGFR3.

\section{References}

[1] Jain RK. Molecular regulation of vessel maturation. Nat Med 2003;9:685-93.

[2] Olsson AK, Dimberg A, Kreuger J, Claesson-Welsh L. VEGF receptor signalling - in control of vascular function. Nat Rev Mol Cell Biol 2006;7:359-71.

[3] Yancopoulos GD, Davis S, Gale NW, Rudge JS, Wiegand SJ, Holash J. Vascular-specific growth factors and blood vessel formation. Nature 2000;407:242-8.

[4] Serini G, Valdembri D, Bussolino F. Integrins and angiogenesis: a sticky business. Exp Cell Res 2006;312:651-8.

[5] Qin J, Vinogradova O, Plow EF. Integrin bidirectional signalling: a molecular view. PLoS Biol 2004;2:0726-9.

[6] Soldi R, Mitola S, Strasly S, Defilippi P, Tarone G, Bussolino F. Role of avb3 integrin in the activation of vascular endothelial growth factor receptor-2. EMBO J 1999;18:734-40.

[7] Borges E, Jan Y, Ruoslahti E. PDGF-receptor-and VEGFreceptor-2 bind to the 3 integrin through its extracellular domain. J Biol Chem 2000;275:39867-73.

[8] Mahabeleshwar GH, Feng W, Phillips DR, Byzova V. Integrin signalling is critical for pathological angiogenesis. J Exp Med 2006;203:2495-507.

[9] Masson-Gadais B, Houle F, Laferriere J, Huot J. Integrin avb3 requirement for VEGFR2-mediated activation of SAPK2/p38 and for Hsp90-dependent phosphorylation of focal adhesion kinase in endothelial cells activated by VEGF. Cell Stress Chaperones 2003;8:37-52.

[10] Serini G, Valdembri D, Zanivan S, Morterra G, Burkhardt C, Caccavari F, et al. Class 3 semaphorins control vascular morphogenesis by inhibiting integrin function. Nature 2003;424: 391-7.

[11] Dardik R, Loscalzo J, Eskaraev R, Inba A. Molecular mechanisms underlying the proangiogenic effect of factor XIII. Arterioscler Thromb Vasc Biol 2005;25:526-32.

[12] Mitola S, Brenchio B, Piccinini M, Tertoolen L, Zammataro L, Breier G, et al. Type I collagen limits VEGFR-2 signalling by a SHP2 protein-tyrosine phosphatase-dependent mechanism 1. Circ Res 2006;98:45-54.

[13] Seo DW, Li H, Guedez L, Wingfield PT, Diaz T, Salloum R, et al. TIMP-2 mediated inhibition of angiogenesis: an MMP-independent mechanism. Cell 2003;114:171-80.

[14] Mattila E, Pellinen T, Nevo J, Vuoriluoto K, Arjonen A, Ivaska J. Negative regulation of EGFR signalling through integrin-alfa1 
beta1-mediated activation of protein tyrosine phosphatase TCPTP. Nat Cell Biol 2005;7:78-85.

[15] Ling Y, Maile LA, Clemmons DR. Tyrosine phosphorylation of the beta3-subunit of the alphaVbeta3 integrin is required for embrane association of the tyrosine phosphatase SHP-2 and its further recruitment to the insulin-like growth factor I receptor. Mol Endocrinol 2003;17:1824-33.
[16] Zhang X, Groopman JE, Wang JF. Extracellular matrix regulates endothelial functions through interaction of VEGFR-3 and integrin a5b1. J Cell Physiol 2005;202:205-14.

[17] Carlson T, Feng Y, Maisonpierre P, Mrksich M, Morla A. Direct cell adhesion to the angiopoietins mediated by integrins. J Biol Chem 2001;278:26516-25.

\section{Genetic variations of Toll-like receptor 9 predispose to systemic lupus erythematosus in Japanese population.}

Systemic lupus erythematosus (SLE) is characterized by dysregulation of autoreactive lymphocytes and antigenpresenting cells. Signaling through Toll-like receptor 9 (TLR9). A mediator of innate immune responses, has a role in activation of dendritic cells and autoreactive B cells. In this study, Kayoko T. et. al. (Ann Rheum Dis 2007; 66: 905-9) asked to investigate whether TLR9 polymorphisms are associated with an increased risk of SLE. DNA samples were obtained from 220 Japanese patients with SLE and 203 controls. The genetic variations of TLR9 were detected by PCR, followed by DNA sequencing. The promoter and enhancer activities of TLR9 were measured by luciferase reporter gene assay. The titers of anti-dsDNA antibodies in sera from control or TLR9deficient mice were analyzed by ELISA. The $\mathrm{G}$ allele at position +1174 (located in intron 1 of TLR9) is closely associated with an increased risk of SLE $(\mathrm{p}=0.029)$. furthermore, patients with SLE tend to have C allele at position - $1486(\mathrm{p}=0.11)$. Both alleles down regulate TLR9 expression by reporter gene assay. TLR9-deficient mice under a C57BL/6 background possess higher titers of anti-dsDNA serum antibodies than control CD57BL/6 mice. These results indicate that the presence of the G allele at position +1174 of TLR9 predisposes humans to an increased risk of SLE. It is speculated that TLR9 normally prevents the development of human SLE.

\section{CD40 Ligand and MHC class expression are essential for human peripheral B cell tolerance.}

Hyper-IgM (HIGM) syndromes are primary immunodeficiencies characterized by defects of class switch recombination and somatic hypermutation. HIGM patients who carry mutations in the CD40-ligand (CD40L) gene expressed by CD4 (+) T cells suffer from recurrent infections and often develop autoimmune disorders. In order to investigate the impact of CD40L-CD40 interactions on human B cell tolerance, Herv M. et. al. (J Exp Med 2007; 204: 1583-93) tested by ELISA the reactivity of recombinant antibodies isolated from single B cells from three CD40L-deficient patients. Antibody characteristics and reactivity from CD40L-deficient new emigrant B cells were similar to those from healthy donors, suggesting that CD40L-CD40 interactions do not regulate central B cell tolerance. In contrast, mature naïve B cells from CD40L-deficient patients expressed a high proportion of autoreactive antibodies, including antinuclear antibodies. Thus, CD40L-CD40 interactions are essential for peripheral B cell tolerance. In addition, a patient with the bare lymphocyte syndrome who could not express MHC II molecules failed to counter select autoreactive mature naïve B cells, suggesting that peripheral B cell tolerance also depends on MHC class II-T cell receptor (TCR) interactions. The decreased frequency of MHC class IIrestricted $\mathrm{CD}(+)$ regulatory $\mathrm{T}$ cells in $\mathrm{CD} 40 \mathrm{~L}-$ deficient patients suggests that these $\mathrm{T}$ cells may mediate peripheral $\mathrm{B}$ cell tolerance through CD40L-CD40 and MHC class II-TCR interactions. 